\title{
Certain subclasses of bi-univalent functions associated with the Hohlov operator
}

\author{
H. M. Srivastava ${ }^{1 *}$, G. Murugusundaramoorthy ${ }^{2}$ and N. Magesh ${ }^{3}$ \\ ${ }^{1}$ Department of Mathematics and Statistics, University of Victoria, Victoria, British Columbia, Canada \\ ${ }^{2}$ School of Advanced Sciences, VIT University, Tamilnadu, India \\ ${ }^{3}$ Post-Graduate and Research Department of Mathematics, Government Arts College for Men, Tamilnadu, India \\ *Corresponding author E-mail: harimsri@math.uvic.ca
}

\begin{abstract}
In this paper, we introduce and investigate two new subclasses of the function class $\Sigma$ of bi-univalent functions defined in the open unit disk, which are associated with the Hohlov operator, that is, a familiar special case of the widely- (and extensively-) investigated Dziok-Srivastava linear operator. Furthermore, we find estimates on the Taylor-Maclaurin coefficients $\left|a_{2}\right|$ and $\left|a_{3}\right|$ for functions in these new subclasses. Several (known or new) consequences of the results are also pointed out.
\end{abstract}

2010 Mathematics Subject Classification: Primary 30C45.

Keywords: Analytic functions, Bi-convex functions, Bi-starlike functions, Bi-univalent functions, Dziok-Srivastava operator, Gaussian hypergeometric function, Hohlov operator, Univalent functions.

\section{Introduction, Definitions and Preliminaries}

Let $\mathcal{A}$ denote the class of functions of the form:

$$
f(z)=z+\sum_{n=2}^{\infty} a_{n} z^{n},
$$

which are analytic in the open unit disk

$$
\mathbb{U}=\{z: z \in \mathbb{C} \text { and }|z|<1\} .
$$

Further, by $\mathcal{S}$ we shall denote the class of all functions in $\mathcal{A}$ which are univalent in $\mathbb{U}$.

The convolution or Hadamard product of two functions $f, h \in \mathcal{A}$ is denoted by $f * h$ and is defined as

$$
(f * h)(z)=z+\sum_{n=2}^{\infty} a_{n} b_{n} z^{n},
$$

where $f(z)$ is given by (1) and $h(z)=z+\sum_{n=2}^{\infty} b_{n} z^{n}$. In terms of the Hadamard product (or convolution), the Dziok-Srivastava linear operator involving the generalized hypergeometric function, was introduced and studied systematically by Dziok and Srivastava [5, 4] and (subsequently) by many other authors. Here, in our present investigation, we recall a familiar convolution operator $\mathcal{I}_{a, b, c}$ due to Hohlov [8,9], which indeed is a very specialized case of the widely- (and extensively-) investigated Dziok-Srivastava operator.

For the complex parameters $a, b$ and $c$ with $c \neq 0,-1,-2,-3, \cdots$, the Gaussian hypergeometric function ${ }_{2} F_{1}(a, b, c ; z)$ is defined as

$$
\begin{aligned}
{ }_{2} F_{1}(a, b, c ; z) & =\sum_{n=0}^{\infty} \frac{(a)_{n}(b)_{n}}{(c)_{n}} \frac{z^{n}}{n !} \\
& =1+\sum_{n=2}^{\infty} \frac{(a)_{n-1}(b)_{n-1}}{(c)_{n-1}} \frac{z^{n-1}}{(n-1) !} \quad(z \in \mathbb{U})
\end{aligned}
$$


where $(\alpha)_{n}$ is the Pochhammer symbol (or the shifted factorial) defined as follows:

$$
(\alpha)_{n}=\frac{\Gamma(\alpha+n)}{\Gamma(\alpha)}= \begin{cases}1 & (n=0) \\ \alpha(\alpha+1)(\alpha+2) \cdots(\alpha+n-1) & (n=1,2,3, \cdots) .\end{cases}
$$

By using the Gaussian hypergeometric function given by (3), Hohlov [8, 9] introduced the familiar convolution operator $\mathcal{I}_{a, b, c}$ as follows:

$$
\begin{aligned}
\mathcal{I}_{a, b ; c} f(z) & =z_{2} F_{1}(a, b, c ; z) * f(z), \\
& =z+\sum_{n=2}^{\infty} \varphi_{n} a_{n} z^{n} \quad(z \in \mathbb{U}),
\end{aligned}
$$

where

$$
\varphi_{n}=\frac{(a)_{n-1}(b)_{n-1}}{(c)_{n-1}(n-1) !} .
$$

Hohlov $[8,9]$ discussed some interesting geometrical properties exhibited by the operator $\mathcal{I}_{a, b ; c}$. The three-parameter family of operators $\mathcal{I}_{a, b ; c}$ contains, as its special cases, most of the known linear integral or differential operators. In particular, if $b=1$ in (5), then $\mathcal{I}_{a, b ; c}$ reduces to the Carlson-Shaffer operator. Similarly, it is easily seen that the Hohlov operator $\mathcal{I}_{a, b ; c}$ is also a generalization of the Ruscheweyh derivative operator as well as the Bernardi-Libera-Livingston operator.

Some of the important and well-investigated subclasses of the univalent function class $\mathcal{S}$ include (for example) the class $\mathcal{S}^{*}(\alpha)$ of starlike functions of order $\alpha$ in $\mathbb{U}$ and the class $\mathcal{K}(\alpha)$ of convex functions of order $\alpha$ in $\mathbb{U}$. It is well known that every function $f \in \mathcal{S}$ has an inverse $f^{-1}$, defined by

$$
f^{-1}(f(z))=z \quad(z \in \mathbb{U})
$$

and

$$
f\left(f^{-1}(w)\right)=w \quad\left(|w|<r_{0}(f) ; r_{0}(f) \geqq \frac{1}{4}\right)
$$

where

$$
f^{-1}(w)=w-a_{2} w^{2}+\left(2 a_{2}^{2}-a_{3}\right) w^{3}-\left(5 a_{2}^{3}-5 a_{2} a_{3}+a_{4}\right) w^{4}+\cdots .
$$

A function $f \in \mathcal{A}$ is said to be bi-univalent in $\mathbb{U}$ if both $f(z)$ and $f^{-1}(z)$ are univalent in $\mathbb{U}$. Let $\Sigma$ denote the class of bi-univalent functions in $\mathbb{U}$ given by (1).

In 1967, Lewin [10] investigated the bi-univalent function class $\Sigma$ and showed that $\left|a_{2}\right|<1.51$. On the other hand, Brannan and Clunie [1] (see also [2, 3, 15]) and Netanyahu [12] made an attempt to introduce various subclasses of the bi-univalent function class $\Sigma$ and obtained non-sharp coefficient estimates on the first two coefficients $\left|a_{2}\right|$ and $\left|a_{3}\right|$ of (1). But the coefficient problem for each of the following Taylor-Maclaurin coefficients:

$$
\left|a_{n}\right| \quad(n \in \mathbb{N} \backslash\{1,2\} ; \mathbb{N}:=\{1,2,3, \cdots\}
$$

is still an open problem. Following Brannan and Taha [3], many researchers (see [6, 7, 11, 14, 16, 17]) have recently introduced and investigated several interesting subclasses of the bi-univalent function class $\Sigma$ and they have found non-sharp estimates on the first two Taylor-Maclaurin coefficients $\left|a_{2}\right|$ and $\left|a_{3}\right|$.

Making use of the Hohlov operator $\mathcal{I}_{a, b ; c}$, we introduce the following two new subclasses of the function class $\Sigma$.

Definition 1.1. A function $f(z)$ given by (1) is said to be in the class $\mathcal{S}_{\Sigma}^{a, b ; c}(\alpha, \lambda)$ if the following conditions are satisfied:

$$
f \in \Sigma \text { and }\left|\arg \left(\frac{z\left(\mathcal{I}_{a, b ; c} f(z)\right)^{\prime}}{(1-\lambda) z+\lambda \mathcal{I}_{a, b ; c} f(z)}\right)\right|<\frac{\alpha \pi}{2} \quad(0<\alpha \leqq 1 ; 0 \leqq \lambda \leqq 1 ; z \in \mathbb{U})
$$

and

$$
\left|\arg \left(\frac{w\left(\mathcal{I}_{a, b ; c} g(w)\right)^{\prime}}{(1-\lambda) w+\lambda \mathcal{I}_{a, b ; c} g(w)}\right)\right|<\frac{\alpha \pi}{2} \quad(0<\alpha \leqq 1 ; 0 \leqq \lambda \leqq 1 ; w \in \mathbb{U}),
$$

where the function $g$ is given by

$$
g(w)=w-a_{2} w^{2}+\left(2 a_{2}^{2}-a_{3}\right) w^{3}-\left(5 a_{2}^{3}-5 a_{2} a_{3}+a_{4}\right) w^{4}+\cdots,
$$

that is, the extension of $f^{-1}$ to $\mathbb{U}$. 
We note that for $\lambda=0, a=c$ and $b=1$, the class $\mathcal{S}_{\Sigma}^{a, b ; c}(\alpha, \lambda)$ reduces to the class $\mathcal{H}_{\Sigma}^{\alpha}$ introduced and studied by Srivastava et al. [14]. Putting $\lambda=1, a=c$ and $b=1$, the class $\mathcal{S}_{\Sigma}^{a, b ; c}(\alpha, \lambda)$ reduces to the class of strongly bi-starlike functions of order $\alpha(0<\alpha \leqq 1)$ and denoted by $\mathcal{S}_{\Sigma}^{*}(\alpha)$.

Definition 1.2. A function $f(z)$ given by (1) is said to be in the class $\mathcal{M}_{\Sigma}^{a, b ; c}(\beta, \lambda)$ if the following conditions are satisfied:

$$
f \in \Sigma \quad \text { and } \quad \Re\left(\frac{z\left(\mathcal{I}_{a, b ; c} f(z)\right)^{\prime}}{(1-\lambda) z+\lambda \mathcal{I}_{a, b ; c} f(z)}\right)>\beta \quad(0 \leqq \beta<1 ; 0 \leqq \lambda \leqq 1 ; z \in \mathbb{U})
$$

and

$$
\Re\left(\frac{w\left(\mathcal{I}_{a, b ; c} g(w)\right)^{\prime}}{(1-\lambda) w+\lambda \mathcal{I}_{a, b ; c} g(w)}\right)>\beta \quad(0 \leqq \beta<1 ; 0 \leqq \lambda \leqq 1 ; w \in \mathbb{U}),
$$

where the function $g$ is given by (10).

It is interesting to note that, for $\lambda=0, a=c$ and $b=1$, the class $\mathcal{M}_{\Sigma}^{a, b ; c}(\beta, \lambda)$ reduces to the class $\mathcal{H}_{\Sigma}^{\beta}$ introduced and studied by Srivastava et al. [14]. Putting $\lambda=1, a=c$ and $b=1$, the class $\mathcal{M}_{\Sigma}^{a, b ; c}(\beta, \lambda)$ reduces to the class of bi-starlike functions of order $\beta(0<\beta \leqq 1)$ and denoted by $\mathcal{S}_{\Sigma}(\beta)$.

The object of the present paper is to find estimates on the coefficients $\left|a_{2}\right|$ and $\left|a_{3}\right|$ for functions in the above-defined subclasses $\mathcal{S}_{\Sigma}^{a, b ; c}(\alpha, \lambda)$ and $\mathcal{M}_{\Sigma}^{a, b ; c}(\alpha, \lambda)$ of the function class $\Sigma$ by employing the techniques used earlier by Srivastava et al. [14].

In order to derive our main results, we shall need the following lemma.

Lemma 1.3. (see [13]) If $h \in \mathcal{P}$, then $\left|c_{k}\right| \leqq 2$ for each $k$, where $\mathcal{P}$ is the family of all functions $h$, analytic in $\mathbb{U}$, for which

$$
\Re\{h(z)\}>0 \quad(z \in \mathbb{U}),
$$

where

$$
h(z)=1+c_{1} z+c_{2} z^{2}+\cdots \quad(z \in \mathbb{U}) .
$$

\section{Coefficient Bounds for the Function Class $\mathcal{S}_{\Sigma}^{a, b ; c}(\alpha, \lambda)$}

We begin by finding the estimates on the coefficients $\left|a_{2}\right|$ and $\left|a_{3}\right|$ for functions in the class $\mathcal{S}_{\Sigma}^{a, b ; c}(\alpha, \lambda)$.

Theorem 2.1. Let the function $f(z)$ given by (1) be in the following class:

$$
\mathcal{S}_{\Sigma}^{a, b ; c}(\alpha, \lambda) \quad(0<\alpha \leqq 1 ; 0 \leqq \lambda \leqq 1) .
$$

Then

$$
\left|a_{2}\right| \leqq \frac{2 \alpha}{\sqrt{\left[2 \alpha\left(\lambda^{2}-2 \lambda\right)+(1-\alpha)(2-\lambda)^{2}\right] \varphi_{2}^{2}+2 \alpha(3-\lambda) \varphi_{3}}}
$$

and

$$
\left|a_{3}\right| \leqq \frac{2 \alpha}{(3-\lambda) \varphi_{3}} .
$$

Proof. It follows from (8) and (9) that

$$
\frac{z\left(\mathcal{I}_{a, b ; c} f(z)\right)^{\prime}}{(1-\lambda) z+\lambda \mathcal{I}_{a, b ; c} f(z)}=[p(z)]^{\alpha}
$$

and

$$
\frac{w\left(\mathcal{I}_{a, b ; c} g(w)\right)^{\prime}}{(1-\lambda) w+\lambda \mathcal{I}_{a, b ; c} g(w)}=[q(w)]^{\alpha},
$$

where $p(z)$ and $q(w)$ in $\wp$ and have the following forms:

$$
p(z)=1+p_{1} z+p_{2} z^{2}+\cdots
$$

and

$$
q(z)=1+q_{1} w+q_{2} w^{2}+\cdots,
$$


respectively. Now, equating the coefficients in (15) and (16), we get

$$
\begin{aligned}
& (2-\lambda) \varphi_{2} a_{2}=\alpha p_{1} \\
& \left(\lambda^{2}-2 \lambda\right) \varphi_{2}^{2} a_{2}^{2}+(3-\lambda) \varphi_{3} a_{3}=\frac{1}{2}\left[\alpha(\alpha-1) p_{1}^{2}+2 \alpha p_{2}\right], \\
& -(2-\lambda) \varphi_{2} a_{2}=\alpha q_{1}
\end{aligned}
$$

and

$$
\left(\lambda^{2}-2 \lambda\right) \varphi_{2}^{2} a_{2}^{2}+(3-\lambda) \varphi_{3}\left(2 a_{2}^{2}-a_{3}\right)=\frac{1}{2}\left[\alpha(\alpha-1) q_{1}^{2}+2 \alpha q_{2}\right] .
$$

From (19) and (21), we find that

$$
a_{2}=\frac{\alpha p_{1}}{(2-\lambda) \varphi_{2}}=\frac{-\alpha q_{1}}{(2-\lambda) \varphi_{2}}
$$

which implies

$$
p_{1}=-q_{1} .
$$

Adding (20) and (22), we obtain

$$
\left[2\left(\lambda^{2}-2 \lambda\right) \varphi_{2}^{2}+2(3-\lambda) \varphi_{3}\right] a_{2}^{2}=\frac{\alpha(\alpha-1)}{2}\left(p_{1}^{2}+q_{1}^{2}\right)+\alpha\left(p_{2}+q_{2}\right) .
$$

Substituting the value of $a_{2}$ from (23) and (24) into (25), we get

$$
p_{1}^{2}=\frac{(2-\lambda)^{2} \varphi_{2}^{2}\left(p_{2}+q_{2}\right)}{\left[2 \alpha\left(\lambda^{2}-2 \lambda\right)+(1-\alpha)(2-\lambda)^{2}\right] \varphi_{2}^{2}+2 \alpha(3-\lambda) \varphi_{3}} .
$$

Applying Lemma 1.3 for the coefficients $p_{2}$ and $q_{2}$, we immediately have

$$
\left|p_{1}\right| \leqq \frac{2(2-\lambda) \varphi_{2}}{\sqrt{\left[2 \alpha\left(\lambda^{2}-2 \lambda\right)+(1-\alpha)(2-\lambda)^{2}\right] \varphi_{2}^{2}+2 \alpha(3-\lambda) \varphi_{3}}}
$$

Thus, (23) gives

$$
\left|a_{2}\right| \leqq \frac{2 \alpha}{\sqrt{\left[2 \alpha\left(\lambda^{2}-2 \lambda\right)+(1-\alpha)(2-\lambda)^{2}\right] \varphi_{2}^{2}+2 \alpha(3-\lambda) \varphi_{3}}} .
$$

This gives the bound on $\left|a_{2}\right|$ as asserted in (13).

Next, in order to find the bound on $\left|a_{3}\right|$, by subtracting (22) from (20), we get

$$
2(3-\lambda) \varphi_{3} a_{3}-2(3-\lambda) \varphi_{3} a_{2}^{2}=\alpha\left(p_{2}-q_{2}\right)+\frac{\alpha(\alpha-1)}{2}\left(p_{1}^{2}-q_{1}^{2}\right) .
$$

It follows from (23), (24) and (28) that

$$
\begin{aligned}
2(3-\lambda) \varphi_{3} a_{3}=\quad\left[\frac{2(3-\lambda) \alpha^{2} \varphi_{3}}{\left[2 \alpha\left(\lambda^{2}-2 \lambda\right)+(1-\alpha)(2-\lambda)^{2}\right] \varphi_{2}^{2}+2 \alpha(3-\lambda) \varphi_{3}}+\alpha\right] p_{2} \\
\quad+\left[\frac{2(3-\lambda) \alpha^{2} \varphi_{3}}{\left[2 \alpha\left(\lambda^{2}-2 \lambda\right)+(1-\alpha)(2-\lambda)^{2}\right] \varphi_{2}^{2}+2 \alpha(3-\lambda) \varphi_{3}}-\alpha\right] q_{2} .
\end{aligned}
$$

Applying Lemma 1.3 once again for the coefficients $p_{2}$ and $q_{2}$, we readily get

$$
\left|a_{3}\right| \leqq \frac{2 \alpha}{(3-\lambda) \varphi_{3}} .
$$

This completes the proof of Theorem 2.1.

Putting $\lambda=0$ in Theorem 2.1, we have the following corollary.

Corollary 2.2. Let the function $f(z)$ given by (1) be in the class $\mathcal{S}_{\Sigma}^{a, b ; c}(\alpha)(0<\alpha \leqq 1)$. Then

$$
\left|a_{2}\right| \leqq \alpha \sqrt{\frac{2}{2(1-\alpha) \varphi_{2}^{2}+3 \alpha \varphi_{3}}}
$$

and

$$
\left|a_{3}\right| \leqq \frac{2 \alpha}{3 \varphi_{3}}
$$


Taking $a=c$ and $b=1$, in Corollary 2.2, we get the following corollary.

Corollary 2.3. Let the function $f(z)$ given by (1) be in the class $\mathcal{H}_{\Sigma}^{\alpha}(0<\alpha \leqq 1)$. Then

$$
\left|a_{2}\right| \leqq \alpha \sqrt{\frac{2}{2+\alpha}}
$$

and

$$
\left|a_{3}\right| \leqq \frac{2 \alpha}{3}
$$

Remark 2.4. The bound on $\left|a_{3}\right|$ in Corollary 2.3 provides an improvement over the result of Srivastava et al. [14].

When $\lambda=1$ in Theorem 2.1, we have the following corollary.

Corollary 2.5. Let the function $f(z)$ given by (1) be in the class $\mathcal{S}_{\Sigma}^{a, b, c}(\alpha, 1)(0<\alpha \leqq 1)$. Then

$$
\left|a_{2}\right| \leqq \frac{2 \alpha}{\sqrt{(1-3 \alpha) \varphi_{2}^{2}+4 \alpha \varphi_{3}}} \quad \text { and } \quad\left|a_{3}\right| \leqq \frac{\alpha}{\varphi_{3}} .
$$

\section{Coefficient Bounds for the Function Class $\mathcal{M}_{\Sigma}^{a, b ; c}(\beta, \lambda)$}

In this section, we find the estimates on the coefficients $\left|a_{2}\right|$ and $\left|a_{3}\right|$ for functions in the class $\mathcal{M}_{\Sigma}^{a, b ; c}(\beta, \lambda)$.

Theorem 3.1. Let the function $f(z)$ given by (1) be in the following class:

$$
\mathcal{M}_{\Sigma}^{a, b ; c}(\beta, \lambda) \quad(0 \leqq \beta<1 ; 0 \leqq \lambda \leqq 1) .
$$

Then

$$
\left|a_{2}\right| \leqq \sqrt{\frac{2(1-\beta)}{\left(\lambda^{2}-2 \lambda\right) \varphi_{2}^{2}+(3-\lambda) \varphi_{3}}}
$$

and

$$
\left|a_{3}\right| \leqq \frac{2(1-\beta)}{(3-\lambda) \varphi_{3}} .
$$

Proof. It follows from (11) and (12) that there exist $p, q \in \wp$ such that

$$
\frac{z\left(\mathcal{I}_{a, b ; c} f(z)\right)^{\prime}}{(1-\lambda) z+\lambda \mathcal{I}_{a, b ; c} f(z)}=\beta+(1-\beta) p(z)
$$

and

$$
\frac{w\left(\mathcal{I}_{a, b ; c} g(w)\right)^{\prime}}{(1-\lambda) w+\lambda \mathcal{I}_{a, b ; c} g(w)}=\beta+(1-\beta) q(w),
$$

where $p(z)$ and $q(w)$ have the forms (17) and (18), respectively. Equating coefficients in (35) and (36), we get

$$
\begin{aligned}
& (2-\lambda) \varphi_{2} a_{2}=(1-\beta) p_{1} \\
& \left(\lambda^{2}-2 \lambda\right) \varphi_{2}^{2} a_{2}^{2}+(3-\lambda) \varphi_{3} a_{3}=(1-\beta) p_{2}, \\
& -(2-\lambda) \varphi_{2} a_{2}=(1-\beta) q_{1}
\end{aligned}
$$

and

$$
\left(\lambda^{2}-2 \lambda\right) \varphi_{2}^{2} a_{2}^{2}+(3-\lambda) \varphi_{3}\left(2 a_{2}^{2}-a_{3}\right)=(1-\beta) q_{2} .
$$

From (37) and (39), we get

$$
a_{2}=\frac{(1-\beta)}{(2-\lambda) \varphi_{2}} p_{1}=\frac{-(1-\beta)}{(2-\lambda) \varphi_{2}} q_{1}
$$

which implies

$$
p_{1}=-q_{1} .
$$


From (38) and (40), we have

$$
\left[2\left(\lambda^{2}-2 \lambda\right)^{2} \varphi_{2}^{2}+2(3-\lambda) \varphi_{3}\right] a_{2}^{2}=(1-\beta)\left(p_{2}+q_{2}\right) .
$$

Also, by using (41) and (43), we obtain

$$
p_{1}^{2}=\frac{(2-\lambda)^{2} \varphi_{2}^{2}\left(p_{2}+q_{2}\right)}{2\left[\left(\lambda^{2}-2 \lambda\right)^{2} \varphi_{2}^{2}+(3-\lambda) \varphi_{3}\right](1-\beta)} .
$$

Applying Lemma 1.3, we get

$$
\left|p_{1}\right| \leqq(2-\lambda) \varphi_{2} \sqrt{\frac{2}{\left[\left(\lambda^{2}-2 \lambda\right)^{2} \varphi_{2}^{2}+(3-\lambda) \varphi_{3}\right](1-\beta)}} .
$$

Again, by applying Lemma 1.3 to (41) and using (45), we immediately find that

$$
\left|a_{2}\right| \leqq \sqrt{\frac{2(1-\beta)}{\left(\lambda^{2}-2 \lambda\right) \varphi_{2}^{2}+(3-\lambda) \varphi_{3}}} .
$$

This gives the bound on $\left|a_{2}\right|$ as asserted in (33).

Next, in order to find the bound on $\left|a_{3}\right|$, by subtracting (40) from (38), we get

$$
2(3-\lambda) \varphi_{3} a_{3}-2(3-\lambda) \varphi_{3} a_{2}^{2}=(1-\beta)\left(p_{2}-q_{2}\right) .
$$

It follows from (43 and 46 that

$$
\begin{gathered}
2(3-\lambda) \varphi_{3} a_{3}=\quad \frac{2(3-\lambda) \varphi_{3}(1-\beta)+\left(\lambda^{2}-2 \lambda\right) \varphi_{2}^{2}(1-\beta)}{\left(\lambda^{2}-2 \lambda\right) \varphi_{2}^{2}+(3-\lambda) \varphi_{3}} p_{2} \\
-\frac{\left(\lambda^{2}-2 \lambda\right) \varphi_{2}^{2}(1-\beta)}{\left(\lambda^{2}-2 \lambda\right) \varphi_{2}^{2}+(3-\lambda) \varphi_{3}} q_{2}
\end{gathered}
$$

Applying Lemma 1.3 once again for the coefficients $p_{2}$ and $q_{2}$, we readily get

$$
\left|a_{3}\right| \leqq \frac{2(1-\beta)}{(3-\lambda) \varphi_{3}} .
$$

This completes the proof of Theorem 3.1.

Putting $\lambda=0$ in Theorem 3.1, we have the following corollary.

Corollary 3.2. Let the function $f(z)$ given by (1) be in the class $\mathcal{M}_{\Sigma}^{a, b, ; c}(\beta)(0 \leqq \beta<1)$. Then

$$
\left|a_{2}\right| \leqq \sqrt{\frac{2(1-\beta)}{3 \varphi_{3}}}
$$

and

$$
\left|a_{3}\right| \leqq \frac{2(1-\beta)}{3 \varphi_{3}}
$$

Remark 3.3. For $a=c$ and $b=1$, the bound on $\left|a_{3}\right|$ in Corollary 3.2 is provides an improvement over the result of Srivastava et al. [14, Theorem2,1191].

When $\lambda=1$ in Theorem 3.1, we get the following corollary for the well-known class $\mathcal{M}_{\Sigma}^{a, b ; c}(\beta, 1)$ of bi-starlike functions of order $\beta$.

Corollary 3.4. Let the function $f(z)$ given by (1) be in the class $\mathcal{S}_{\Sigma}^{a, b ; c}(\beta)(0 \leqq \beta<1)$. Then

$$
\left|a_{2}\right| \leqq \sqrt{\frac{2-2 \beta}{2 \varphi_{3}-\varphi_{2}^{2}}} \quad \text { and } \quad\left|a_{3}\right| \leqq \frac{1-\beta}{\varphi_{3}} .
$$

Remark 3.5. Various other interesting corollaries and consequences of our main results (which are asserted by Theorems 2.1 and 3.1 above) can be derived similarly. The details involved may be left as an exercise for the interested reader. 


\section{References}

[1] D.A. Brannan and J.G. Clunie (Editors), Aspects of Contemporary Complex Analysis, Academic Press, London, 1980.

$[2]$ D. A. Brannan, J. Clunie and W. E. Kirwan, Coefficient estimates for a class of star-like functions, Canad. J. Math. 22 (1970), 476-485.

[3] D. A. Brannan and T. S. Taha, On some classes of bi-univalent functions, Studia Univ. Babeş-Bolyai Math. 31 (2) (1986), 70-77.

[4] J. Dziok and H. M. Srivastava, Classes of analytic functions associated with the generalized hypergeometric function, Appl. Math. Comput. 103 (1999), 1-13.

[5] J. Dziok and H. M. Srivastava, Certain subclasses of analytic functions associated with the generalized hypergeometric function, Intergral Transforms Spec. Funct. 14 (2003), 7-18.

[6] B. A. Frasin and M. K. Aouf, New subclasses of bi-univalent functions, Appl. Math. Lett. 24 (2011), $1569-1573$.

[7] T. Hayami and S. Owa, Coefficient bounds for bi-univalent functions, Pan Amer. Math. J. 22 (4) (2012), 15-26.

[8] Yu. E. Hohlov, Convolution operators that preserve univalent functions, Ukrain. Mat. Zh. 37 (1985), $220-226$.

[9] Yu. E. Hohlov, Hadamard convolutions, hypergeometric functions and linear operators in the class of univalent functions, Dokl. Akad. Nauk Ukrain. SSR Ser. A 1984 (7) (1984), 25-27.

[10] M. Lewin, On a coefficient problem for bi-univalent functions, Proc. Amer. Math. Soc. 18 (1967), 63-68.

[11] X.-F. Li and A.-P. Wang, Two new subclasses of bi-univalent functions, Internat. Math. Forum 7 (2012), 1495-1504.

[12] E. Netanyahu, The minimal distance of the image boundary from the origin and the second coefficient of a univalent function in $z<1$, Arch. Rational Mech. Anal. 32 (1969), 100-112.

[13] C. Pommerenke, Univalent Functions, Vandenhoeck \& Ruprecht, Göttingen, 1975.

[14] H. M. Srivastava, A. K. Mishra and P. Gochhayat, Certain subclasses of analytic and bi-univalent functions, Appl. Math. Lett. 23 (2010), 1188-1192.

[15] T. S. Taha, Topics in Univalent Function Theory, Ph.D. Thesis, University of London, 1981.

[16] Q.-H. Xu, Y.-C. Gui and H. M. Srivastava, Coefficient estimates for a certain subclass of analytic and bi-univalent functions, Appl. Math. Lett. 25 (2012), 990-994.

[17] Q.-H. Xu, H.-G. Xiao and H. M. Srivastava, A certain general subclass of analytic and bi-univalent functions and associated coefficient estimate problems, Appl. Math. Comput. 218 (2012), 11461-11465. 